\title{
On weighted $G$-Banach frames in Banach spaces
}

\author{
GHANSHYAM SINGH RATHORE AND TRIPTI MITTAL ${ }^{\dagger}$
}

\author{
Date of Receiving : $\quad 13.02 .2018$ \\ Date of Revision : $\quad 09.04 .2018$ \\ Date of Acceptance : 10.04 .2018
}

\begin{abstract}
In the present paper, we introduce and study weighted $g$-Banach frames in Banach spaces. Examples and counter examples to distinguish various types of weighted $g$-Banach frames in Banach spaces have been given. A necessary and sufficient condition for a weighted $g$-Banach frame to be an exact has been given. Also, the construction of weighted $g$-Banach frames from bounded linear operator has been discussed. Finally, we study the finite sum of weighted $g$-Banach frames and give a sufficient condition for the finite sum of weighted $g$-Banach frames to be a weighted $g$-Banach frame.
\end{abstract}

\section{Introduction}

Frames are main tools for use in signal processing, image processing, data compression and sampling theory etc. Today even more uses are being found for the theory such as optics, filter banks, signal detection as well as study of Besov spaces, Banach space theory etc. Frames for Hilbert spaces were introduced by Duffin and Schaeffer[14]. Later, in 1986, Daubechies, Grossmann and Meyer [13] reintroduced frames and found a new application to wavelet and Gabor transforms. For a nice introduction to frames, one may refer [6].

Coifman and Weiss [12] introduced the notion of atomic decomposition for function spaces. Feichtinger and Gröchening [16] extended the notion of atomic decomposition to Banach spaces. Gröchening [18] introduced a more general concept for Banach spaces called Banach frame. He gave the following definition of a Banach frame:

Definition 1.1. ([18]) Let $\mathcal{X}$ be a Banach space and $\mathcal{X}_{d_{1}}$ an associated Banach space of scalar-valued sequences indexed by $\mathbb{N}$. Let $\left\{f_{n}\right\} \subset \mathcal{X}^{*}$ and $S: \mathcal{X}_{d_{1}} \rightarrow \mathcal{X}$ be given. Then the pair $\left(\left\{f_{n}\right\}, S\right)$ is called a Banach frame for $\mathcal{X}$ with respect to $\mathcal{X}_{d_{1}}$, if

(1) $\left\{f_{n}(x)\right\} \in \mathcal{X}_{d_{1}}$, for each $x \in \mathcal{X}$.

2010 Mathematics Subject Classification. 42A38, 42C15, 46B15, 41A58.

Key words and phrases. Frame, Banach frame, $g$-frame, $g$-Banach frame.

The authors are thankful to the referee(s) for their valuable time and suggestions towards the improvement of this paper.

Communicated by: Asghar Rahimi

${ }^{\dagger}$ Corresponding author. 